\title{
Network Degeneracy and the Dynamics of Task Switching in the Feeding Circuit in Aplysia
}

\author{
Yanqing Wang, -Klaudiusz R. Weiss, and Elizabeth C. Cropper \\ Department of Neuroscience and Friedman Brain Institute, Icahn School of Medicine at Mount Sinai, New York, New York 10029
}

The characteristics of a network are determined by parameters that describe the intrinsic properties of the component neurons and their synapses. Degeneracy occurs when more than one set of parameters produces the same (or very similar) output. It is not clear whether network degeneracy impacts network function or is simply a reflection of the fact that, although it is important for a network to be able to generate a particular output, it is not important how this is achieved. We address this issue in the feeding network of the mollusc Aplysia. In this system, there are two stimulation paradigms that generate egestive motor programs: repetition priming and positive biasing. We demonstrate that circuit parameters differ in the 2 cases (e.g., egestive repetition priming requires activity in an interneuron, B20, which is not essential for positive biasing). We show that degeneracy has consequences for task switching. If egestive repetition priming is immediately followed by stimulation of an ingestive input to the feeding central pattern generator, the first few cycles of activity are egestive (not ingestive). In this situation, there is a task switch cost. This "cost" is in part due to the potentiating effect of egestive repetition priming on B20. In contrast, there is no switch cost after positive biasing. Stimulation of the ingestive central pattern generator input immediately triggers ingestive activity. Our results indicate that the mechanisms used to pattern activity can impact network function in that they can determine how readily a network can switch from one configuration to another.

Key words: central pattern generator; invertebrate; mollusc; motor program

Significance Statement

A particular pattern of neural activity can be generated by more than one set of circuit parameters. How or whether this impacts network function is unclear. We address this issue in the feeding network of Aplysia and demonstrate that degeneracy in network function can have consequences for task switching. Namely, we show that, when egestive activity is generated via one set of circuit modifications, an immediate switch to ingestive activity is not possible. In contrast, rapid transitions to ingestive activity are possible if egestive activity is generated via a different set of circuit modifications.

\section{Introduction}

It has become apparent that a particular pattern of neural activity can be generated by more than one set of circuit parameters. This has been most extensively documented in the crustacean stomatogastric nervous system (Golowasch, 2014; Marder et al., 2015). Further, it has been reported in the network that generates the leech heartbeat (Norris et al., 2007; Roffman et al., 2012; Lamb and Calabrese, 2013), and in more than one behavioral context in Caenorhabditis elegans (Beverly et al., 2011; Trojanowski et al., 2014). It is tempting to speculate that such circuit degeneracy is simply a byproduct of the fact that evolution acts at the level of

\footnotetext{
Received June 21, 2019; revised Sept. 3, 2019; accepted Sept. 16, 2019.

Author contributions: Y.W., K.R.W., and E.C.C. designed research; Y.W. performed research; Y.W. analyzed data;

Y.W. wrote the first draft of the paper; K.R.W. and E.C.C. edited the paper; E.C.C. wrote the paper.

This work was supported by the National Institutes of Health Grants NS066587 and NS070583.

The authors declare no competing financial interests.

Correspondence should be addressed to Elizabeth C. Cropper at elizabeth.cropper@gmail.com.

https://doi.org/10.1523/JNEUROSCI.1454-19.2019

Copyright $\odot 2019$ the authors
}

overall network performance and not at the level of the properties of individual neurons. In other words, as long as the network can produce the correct output, it does not matter how this is accomplished. Currently, this idea remains virtually unchallenged.

In this report, we study network degeneracy in a circuit that generates more than one output. Namely, we study a feeding circuit that generates ingestive and egestive motor programs. Under physiological conditions, task switching occurs in this circuit. For example, a bout of ingestive activity can be followed by a bout of egestive activity and vice versa (e.g., Proekt et al., 2008). We sought to determine whether there could be a relationship between how motor programs are patterned and how readily task switching can occur. In particular, we sought to determine whether a task switch that readily occurs when egestive activity is patterned using one set of circuit parameters less readily occurs when egestive activity is patterned using an alternative set of parameters.

Our experiments are conducted in the feeding network of the mollusc Aplysia and focus on the neural control of the radula, a 
tongue-like structure that is used to grasp food and pull it into the buccal cavity. Ingestive radula movements pull food into the buccal cavity, whereas egestive movements push it out (Kupfermann, 1974). Previous investigators studying the feeding network have identified radula motor neuron activity that can be used to classify feeding motor programs in the isolated nervous system (e.g., Morton and Chiel, 1993a,b). Further, inputs to the feeding central pattern generator $(\mathrm{CPG})$ that can be used to trigger ingestive and egestive motor programs have been identified (see Fig. 1A) (e.g., Rosen et al., 1991; Church and Lloyd, 1994; Sánchez and Kirk, 2001; Morgan et al., 2002; Proekt et al., 2004; Dacks and Weiss, 2013).

Prior research has demonstrated that egestive motor programs can be triggered using two different stimulation paradigms (see Fig. $1 B$ vs Fig. 1C) (Proekt et al., 2004; Dacks and Weiss, 2013). Previous data suggest that the mechanisms used to pattern motor activity are different in the two cases. One goal of this study was to determine whether this is the case. Second, previous work has established that, when egestive activity is generated using one of the two stimulation paradigms, it is not possible to immediately switch tasks (i.e., switch to an ingestive motor program) (Proekt et al., 2004). In this situation, there is a task switch "cost" (i.e., there is an increase in the amount of time required for the initiation of an ingestive response). A second goal of this study was to determine whether this task switch cost is always observed, or whether it can be avoided if egestive activity is patterned via an alternative set of circuit parameters. Thus, we sought to determine whether network degeneracy can have consequences for task switching.

\section{Materials and Methods}

Animals. Adult sea slugs (Aplysia california) were purchased from Marinus Scientific and maintained in artificial seawater (Instant Ocean) at $14^{\circ} \mathrm{C}-16^{\circ} \mathrm{C}$. Aplysia are hermaphrodites, and are therefore both male and female. Animals weighing 150-200 g were anesthetized by injection of $75-100 \mathrm{ml}$ isotonic (i.e., $337 \mathrm{~mm} \mathrm{MgCl}_{2}$ ). Cerebral and buccal ganglia were dissected out with the cerebral-buccal connectives, esophageal, and buccal nerves intact. The connective tissue surrounding ganglia was removed while ganglia were bathed in a solution containing $50 \%$ artificial seawater (in mM as follows: $60 \mathrm{NaCl}, 10 \mathrm{KCl}, 55 \mathrm{MgCl}_{2}, 11 \mathrm{CaCl}_{2}$, and 10 HEPES buffer, $\mathrm{pH} 7.6$ ) and $50 \%$ isotonic $\mathrm{MgCl}_{2}$. Desheathed ganglia were then superfused with artificial seawater at $0.3 \mathrm{ml} / \mathrm{min}$ and maintained at $\sim 15^{\circ} \mathrm{C}-17^{\circ} \mathrm{C}$.

Cell identification. Neurons were identified based on their location, size, and electrophysiological and morphological characteristics. Neurons recorded from in these experiments are cerebral-buccal interneuron 2 (CBI-2), B8, B20, B40, and B65. CBI-2 is a command-like neuron that triggers ingestive motor programs (Rosen et al., 1991). It is located in the M-cluster of the cerebral ganglion (Rosen et al., 1991; Hurwitz et al., 1999). B8 is a radula closer motor neuron, and it is located on the ventrolateral surface of the buccal ganglion (Morton and Chiel, 1993a,b). $\mathrm{B} 20$ and $\mathrm{B} 65$ are protraction interneurons that promote egestive activity (Jing and Weiss, 2001; Dacks and Weiss, 2013). B20 is located on the rostral surface of the buccal ganglion (Jing and Weiss, 2001). B65 is located on the caudal surface of the buccal ganglion at the intersection of the axonal bundles from buccal nerve one and the esophageal nerve (EN) (Kabotyanski et al., 1998). B40 is an interneuron that promotes ingestive activity (Jing and Weiss, 2002). It is located close to, usually lateral to, B65.

Electrophysiological recordings. Intracellular recordings were performed using glass micropipettes filled with a $0.6 \mathrm{M} \mathrm{K}_{2} \mathrm{SO}_{4}$ and $60 \mathrm{~mm}$ $\mathrm{KCl}$ electrolyte solution. Electrodes were fabricated using a Flaming/ Brown micropipette puller (Sutter Instrument) to yield a final resistance of 6-10 $\mathrm{M} \Omega$. Electrodes were held in HS-2A headstages (Molecular Devices) connected to AxoClamp 2B amplifiers (Molecular Devices). Extracellular nerve recordings were obtained from the EN and I2 nerve using suction electrodes fabricated by aspirating nerves into polyethylene tubing. Both intracellular and extracellular signals were amplified by a CyberAmp 380 (Molecular Devices) and digitized using a Digidata 1320A (Molecular Devices). Data were acquired using AxoScope software (Molecular Devices).

Classification of feeding motor programs. Feeding behaviors in Aplysia can be classified as egestive or ingestive (Kupfermann, 1974; Morton and Chiel, 1993a, b). In both cases, the radula (food-grasping organ) initially protracts. Subsequently, the radula retracts. Behaviors differ in the relative timing of radula opening and closing. In ingestion, the radula is open during protraction and closed during retraction (Morton and Chiel, 1993a, b). This pulls food into the mouth. In egestion, the radula is open during retraction and closed during protraction. This pushes food out (Morton and Chiel, 1993a,b).

In vitro, motor activity was classified as has been previously described (Morton and Chiel, 1993a, b; Jing and Weiss, 2001, 2002; Jing et al., 2004; Proekt et al., 2004, 2007; Friedman and Weiss, 2010). The protraction phase of the motor program was monitored by recording from the I2 nerve, which contains the axons of protraction motor neurons (Hurwitz and Susswein, 1996; Nargeot et al., 1999a, b; Morgan et al., 2000; Jing and Weiss, 2001, 2002). Retraction was defined as the phase of the motor program in which high-frequency, large-amplitude units were recorded in the EN after I2 nerve activity (Morton and Chiel, 1993a, b; Nargeot et al., 1999b). Radula closing was monitored by recording from the B8 motor neurons (Morton and Chiel, 1993a, b). Activity was classified as ingestive when the B8 firing frequency during protraction was $<3.5 \mathrm{~Hz}$, the firing frequency during retraction was $>4.5 \mathrm{~Hz}$, and the ratio of the two numbers was $<0.65$ (Morgan et al., 2000, 2002). Activity was classified as egestive when the B8 firing frequency during protraction was $>3.5 \mathrm{~Hz}$, the firing frequency during retraction was $<2.5 \mathrm{~Hz}$, and the ratio of the two numbers was $>2.0$ (Morgan et al., 2000, 2002). Motor activity was considered intermediate when B8 fired at a low frequency during both protraction and retraction (Proekt et al., 2004; Dacks and Weiss, 2013).

Induction of ingestive and egestive motor programs. Ingestive motor programs were triggered using $\mathrm{CBI}-2$, which receives excitatory input from sensory neurons activated by food (Rosen et al., 1991). CBI-2 was intracellularly stimulated during the protraction phase of the motor program at $9 \mathrm{~Hz}$ with brief $(15 \mathrm{~ms})$ current pulses to elicit one-for-one action potentials (e.g., Friedman and Weiss, 2010). Single cycles of motor activity triggered in this manner most commonly had intermediate characteristics (as has been previously reported) (Proekt et al., 2004). Activity became ingestive when we "primed" preparations by repeatedly inducing motor activity with a relatively short intercycle interval (Proekt et al., 2004). More specifically, in most experiments, we stimulated CBI-2 so that 10 cycles of motor activity were triggered with $30 \mathrm{~s}$ between the termination of the retraction phase of one cycle and the initiation of the protraction phase of the following cycle (Friedman and Weiss, 2010).

Egestive motor programs were triggered via extracellular stimulation of a branch of the EN that contains processes of sensory neurons innervating the gut (Kuslansky et al., 1987). We used two stimulation protocols: egestive repetition priming (Proekt et al., 2004, 2007) and positive biasing (see Fig. $1 B$ vs Fig. 1C) (Dacks and Weiss, 2013). To induce egestive repetition priming, the EN was stimulated continuously with $4-7 \mathrm{~V}, 3 \mathrm{~ms}$ pulses at $2 \mathrm{~Hz}$ for $2 \mathrm{~min}$. The pulse amplitude was adjusted to elicit five successive cycles of activity (see Fig. 1B) (Proekt et al., 2004; Friedman et al., 2009; Siniscalchi et al., 2016). Programs were triggered in rested preparations. In positive biasing experiments, the EN was stimulated in the same manner, but stimulation began $30 \mathrm{~s}$ after CBI-2induced ingestive priming (see Fig. 1C) (Dacks and Weiss, 2013).

Voltage-clamp determination of currents induced during motor programs. Two-electrode voltage-clamp techniques were used to measure currents induced in B8 during motor programs. Phasic (presumably synaptic) currents were evaluated by measuring the total inward current induced in B8 during protraction and subtracting any tonic current that was present (Perkins et al., 2018). Neurons were held at $\sim-60 \mathrm{mV}$ (the normal resting potential of B8).

Excitability. To measure B40 excitability, action potentials were elicited by injecting 2-4 nA, 4 s constant current depolarizing pulses every $30 \mathrm{~s}$. The specific amount of current injected in an individual experiment 
was selected to generate $\sim 24$ spikes under control conditions (before motor programs were triggered in CBI-2). Four stable responses were obtained before each experimental manipulation (i.e., before CBI-2 stimulation). The control excitability was calculated by averaging the number of spikes triggered during these four trials.

Statistics. Data were analyzed in Clampfit and Spike 2 (Cambridge Electronic Design) and organized in Excel. All data were plotted and analyzed in Prism 6 (GraphPad Software). Error bars indicate SEMs, and the significance level was set at $p<0.05$. When one-way ANOVA tests indicated significant effects, individual comparisons were calculated with a Tukey post hoc. When two-way ANOVA tests indicated significant effects, individual comparisons were calculated with Sidak's multiple comparisons. Throughout the results, $n$ refers to the number of preparations.

\section{Results}

Two paradigms that generate egestive motor programs in the feeding circuit of Aplysia

There are two stimulation paradigms that can be used to induce egestive motor programs in the feeding network of Aplysia. In both cases, a branch of the EN that contains the processes of sensory neurons innervating the gut is stimulated (Fig. 1A) (Kuslansky et al., 1987). With one paradigm, motor activity is triggered in a previously quiescent preparation (Fig. 1B). In this situation, the first cycle of motor activity that is generated is poorly defined in that antagonistic motor neurons are coactive. This type of activity is referred to as having intermediate characteristics. As cycles of activity are repeatedly generated, however, phase relationships progressively change (Proekt et al., 2004). This paradigm is referred to as "egestive repetition priming," referring to the fact that as motor activity is repeatedly induced it becomes more and more well defined as egestive. Second, Dacks and Weiss (2013) have demonstrated that, somewhat surprisingly, activity is immediately egestive if the EN is stimulated after a period of ingestive activity (Fig. 1C). This paradigm has been referred to as "positive biasing," referring to the fact that a bout of ingestive activity promotes the subsequent induction of egestive activity.

In previous experiments that studied positive biasing, ingestive activity was induced by repeated stimulation of the command-like neuron CBI-2 followed by one or two cycles of motor activity triggered by stimulating the EN (Fig. 1C) (Dacks and Weiss, 2013). In most cases, only a single cycle of EN induced activity was analyzed. Consequently, these results did not indicate whether repeated activity induced after CBI-2 stimulation would remain egestive. To determine whether it does, we triggered motor programs using CBI-2, induced multiple cycles of activity using the $\mathrm{EN}$, and analyzed the B8 firing frequency during both protraction and retraction (Fig. 2A). All cycles were egestive, and there was no significant change in the $\mathrm{B} 8$ firing frequency as activity progressed (Fig. $2 B, C 1, C 2$ : for protraction, one-way ANOVA; $F_{(3,16)}=1.101, p=$ $0.3776, n=5$ : for retraction, one-way $\operatorname{ANOVA} ; F_{(3,16)}=0.8, p=$ $0.5118, n=5)$.

\section{Is there degeneracy in the feeding circuit of Aplysia?}

One goal of these experiments was to determine whether circuit mechanisms that pattern egestive activity during egestive repetition priming are different from those that pattern activity during the generation of a positively biased response.

\section{Synaptic currents are increased during egestive repetition priming}

When activity becomes egestive, there is an increase in the firing frequency of the $\mathrm{B} 8$ radula closer motor neurons during the protraction phase of motor programs (radula closing during pro-
A

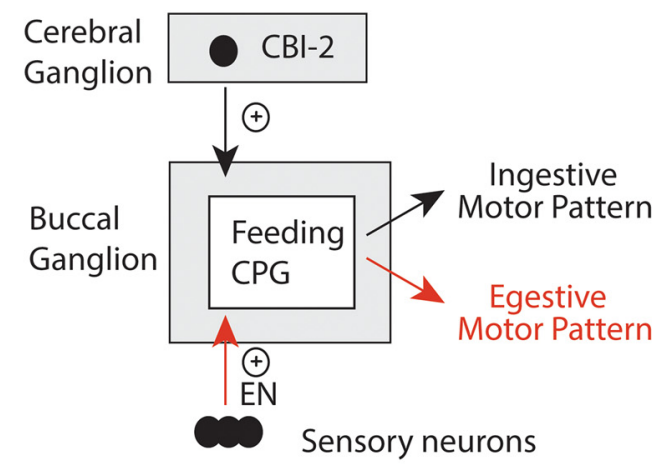

B egestive repetition priming

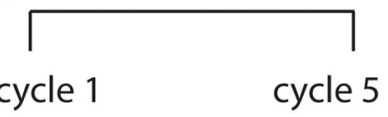

EN

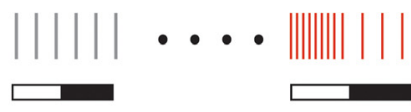

negative biasing

$\mathrm{CBI}-2$

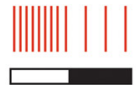

C

positive biasing

EN

ingestive repetition priming
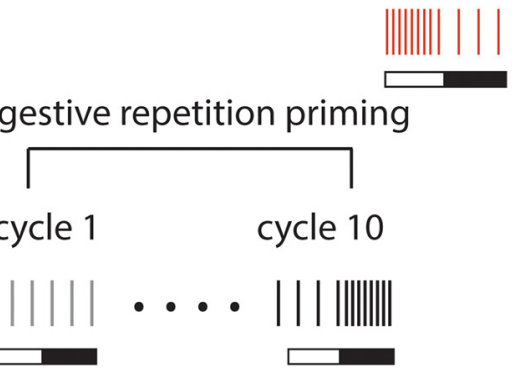

Figure 1. A, Ingestive and egestive inputs to the feeding CPG. Ingestive motor programs can be triggered by stimulating the command-like neuron (BI-2. Egestive motor programs can be triggered by stimulating the EN. $B$, Cycles of motor activity triggered by stimulating the EN and $\mathrm{CBI}-2$. Lines indicate spikes in the radula closer motor neuron B8. Bars represent the two cycles of the motor program. Open bar represents protraction. Closed bar represents retraction. Activity is egestive when B8 fires at a relatively high frequency during protraction and is ingestive when $B 8$ fires at a relatively high frequency during retraction. When motor programs are evoked by stimulating the $\mathrm{EN}$, the first cycle of activity has intermediate characteristics (gray). With repeated EN stimulation, activity becomes egestive (i.e., egestive repetition priming occurs; red). If a cycle of motor activity is triggered by stimulating (BI-2 after egestive priming, negative biasing is observed (i.e., activity is egestive). $\boldsymbol{C}$, When motor programs are evoked by stimulating $\mathrm{CBI}-2$, the first cycle of activity also has intermediate characteristics (gray). With repeated (BI-2 stimulation, activity becomes ingestive (black). If a cycle of motor activity is then triggered by stimulating the $\mathrm{EN}$, positive biasing is observed (i.e., activity is egestive).

traction pushes food out of the buccal cavity) (Morton and Chiel, 1993a,b). In principle, a change in firing frequency can result from either an increase in excitability or increased synaptic input. When egestive activity is induced using the positive biasing paradigm, the change in B8 activity results from increased synaptic input from a protraction phase interneuron (Dacks and Weiss, 2013). We sought to determine whether the same is likely to be true for egestive repetition priming. To accomplish this, we induced motor programs by stimulating the EN (Fig. 3A1) and recorded from one of the four B8 neurons under current-clamp conditions (Fig. 3A2, top) and a second B8 under voltage-clamp conditions (Fig. 3A2, middle). We used the current-clamp recording to verify that egestive repetition priming occurred; that is, 
there was a significant increase in the protraction phase firing frequency of $\mathrm{B} 8$ when motor programs were repeatedly induced (Fig. 3A2,A3: $t_{(5)}=8.56, p=$ 0.0004 , paired $t$ test; $n=6$ ). We used the voltage-clamp recording to monitor corresponding changes in the magnitude of currents. In particular, we sought to determine whether there was an increase in the peak amplitude of the inward current recorded during the protraction phase of the motor program. This current did increase (Fig. $3 A 2, A 4: t_{(5)}=6.30, p=$ 0.0015 , paired $t$ test; $n=6$ ). Similar results were obtained when the change in the protraction phase current was recorded after a positively biased response was generated (Fig. 3B1,B2,B4: $t_{(4)}=4.54, p=$ 0.0105 , paired $t$ test; $n=5$ ). Indeed, the mean protraction phase current recorded after egestive repetition priming was $-6.87 \mathrm{nA}$, and the mean protraction phase current recorded during the positively biased response was $-6.93 \mathrm{nA}\left(t_{(9)}=0.6, p=\right.$ $0.9568)$. These data suggest that egestive repetition priming, like positive biasing, is at least partially mediated by an increase in synaptic input to B8.

An interneuron essential for generating a positively biased response (B65) is not essential for egestive repetition priming

A further question, however, is whether the source of synaptic input is the same under the two conditions (egestive repetition priming vs positive biasing). B65 is an identified protraction phase interneuron that is essential for generating a positively biased response (i.e., when it is hyperpolarized, a positively biased response is not generated) (Dacks and Weiss, 2013). To determine whether $\mathrm{B} 65$ is essential for egestive repetition priming, we triggered motor programs using the $\mathrm{EN}$ and initially induced egestive priming under control conditions (Fig. 4A1). We then attempted to induce egestive repetition priming with $\mathrm{B} 65$ bilaterally hyperpolarized, either during the fifth (last) cycle of motor activity (Fig. 4A2), or throughout all five cycles of the motor program (Fig. 4A3). Neither manipulation had an effect on the B8 firing frequency during protraction (i.e., egestiveness of activity) (Fig. 4B: one-way ANOVA; no differences among the three groups during the last cycle; $\left.F_{(2,15)}=1.57 ; p=0.24 ; n=8\right)$. These data indicate that positive biasing and egestive repetition priming differ in that activity in $\mathrm{B} 65$ is essential for generating a positively biased response, whereas it is not essential for egestive repetition priming.

The interneuron B20 is essential for egestive repetition priming but not for generating a positively biased response To identify an interneuron that is essential for egestive repetition priming, we focused on B20. B20 is a second identified protraction interneuron that promotes egestive activity (Jing and Weiss, 2001) and is activated when programs are triggered by the EN (Proekt et al., 2007). We therefore conducted experiments in which we triggered programs using the EN under control conditions (Fig. 5A1), and with B20 hyperpolarized, either during the fifth (last) cycle of the motor program (Fig. 5A2) or continuously
(Fig. 5A3). Both manipulations had a significant effect on the $\mathrm{B} 8$ firing frequency during protraction (Fig. $5 B$ : one-way ANOVA comparing the three groups during the last cycle; $F_{(2,17)}=25.8$; $p=0.00007 ; n=7)$. These data indicate that, although $\mathrm{B} 65$ is not essential for egestive repetition priming, a second protraction interneuron (B20) is. Together with previous work, this suggests that there is degeneracy in the way that egestive activity is patterned in that the increase in the B8 firing frequency observed when a positively biased response is generated is primarily mediated by input from B65, whereas the increase in B8 activity during egestive repetition priming is primarily mediated by input from B20.

However, the previous study that demonstrated that B65 is essential for generating a positively biased response did not examine the role of B20 in this phenomenon. Since there is an excitatory synaptic connection between B65 and B20 (Jing and Weiss, 2001), a B65 manipulation could impact B20 activity. To determine whether hyperpolarization of B20 does impact the generation of a positively biased response, we began experiments by inducing egestive repetition priming to characterize egestive activity (Fig. 6A1). We then generated egestive activity using the positive biasing paradigm under control conditions (Fig. 6A2) and with hyperpolarization of B20 (Fig. 6A3). When B20 was hyperpolarized $60 \mathrm{~s}$ after stimulation of CBI-2 (the time point previously used to evaluate the role of B65) (Dacks and Weiss, 2013), B20 hyperpolarization had no effect (Fig. 6A3,B: $t_{(3)}=$ $0.20, p=0.8554$, paired $t$ test; $n=4)$. In contrast, when the same type of experiment was performed with B65 hyperpolarization, there was a significant decrease in the protraction phase B8 firing frequency (Fig. 6C: $t_{(4)}=5.08, p=0.0071$, paired $t$ test; $n=5$ ).

To summarize, activity becomes egestive when there is increased activity in the $\mathrm{B} 8$ radula closer motor neurons during the 
A 1. EN $\frac{2 \text { min }}{\frac{1}{1}}$
2.

B8 (V)

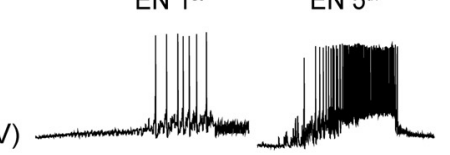

B8 (I)

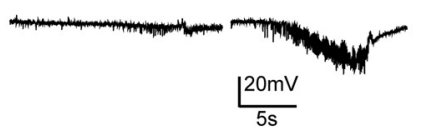

12

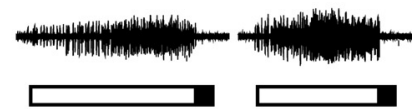

3.

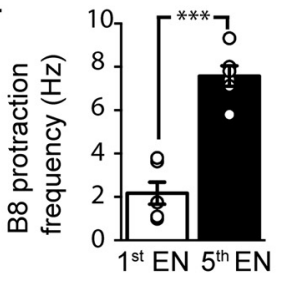

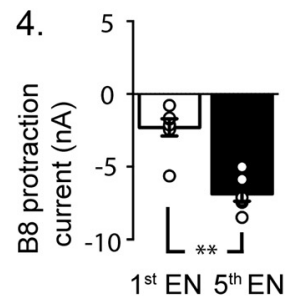

B 1 .

EN

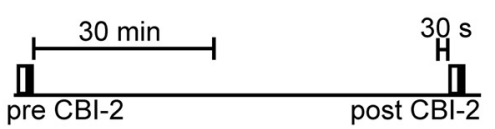

$\mathrm{CBI}-2$

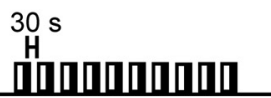

2.
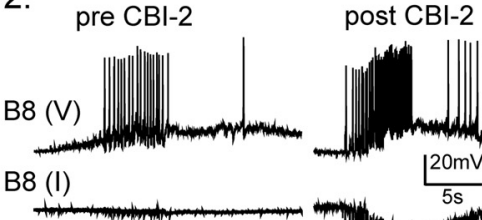

3.

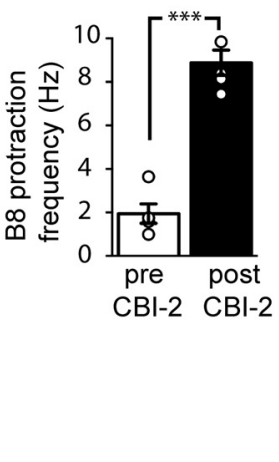

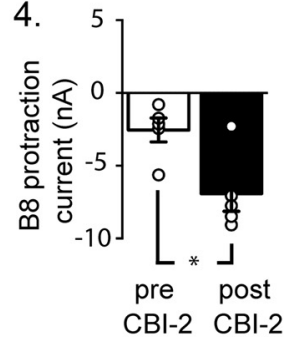

Figure 3. Synaptic currents are increased in B8 during both egestive repetition priming and positive biasing. A1, Egestive repetition priming was induced by stimulating the EN for 2 min, which elicited 5 cycles of motor activity. $\mathbf{A 2}$, Recordings made during the first cycle (EN first) and the fifth cycle (EN fifth). Intracellular recordings were obtained from two B8 neurons: one under current-clamp conditions (top trace, V) and one under voltage-clamp conditions (middle, I). A3, The B8 firing frequency during the protraction phase of the first (white bar) and fifth (black bar) cycle. Activity became egestive; that is, the firing frequency during the fifth cycle was higher than the frequency during the first cycle $(n=6)$. $A 4$, Mean synaptic currents recorded during the protraction phase of the first and fifth cycles of activity. The peak current recorded during the fifth cycle is larger $(n=6)$. B1, Protocol used in positive biasing experiments. A control cycle of activity was triggered by stimulating the EN (pre-CBI-2). Thirty minutes later, positive biasing was induced by stimulating the EN 30 s after ingestive priming (post-CBI-2). B2, Recordings made before and after CBI-2 stimulation. B3, The B8 firing frequency during protraction before (white bar) and after (black bar) CBI-2 stimulation. Activity became egestive; that is, the B8 firing frequency after CBI-2 stimulation was higher than the frequency before (BI-2 stimulation $(n=5)$. B4, Mean synaptic currents recorded during protraction before and after $\mathrm{CBI}-2$ stimulation. The peak current after $\mathrm{CBI}-2$ stimulation is larger $(n=5) .{ }^{*} p<0.05,{ }^{* *} p<0.01,{ }^{* * *} p<0.001$.

\section{A 1. no hyperpolarization}

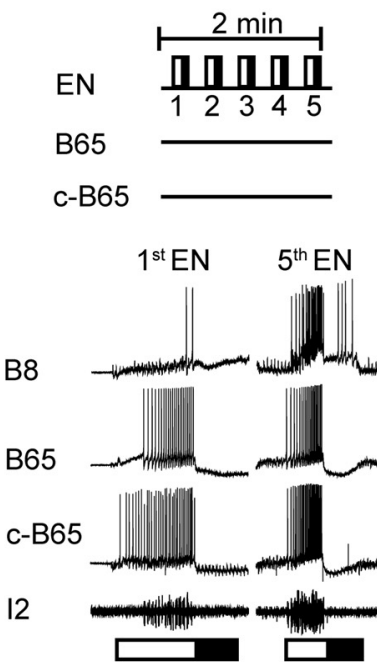

2. hyperpolarization during 5th cycle

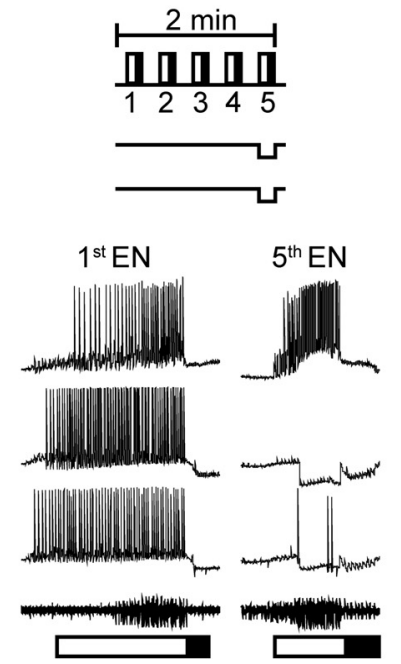

\section{3. hyperpolarization} during all cycles
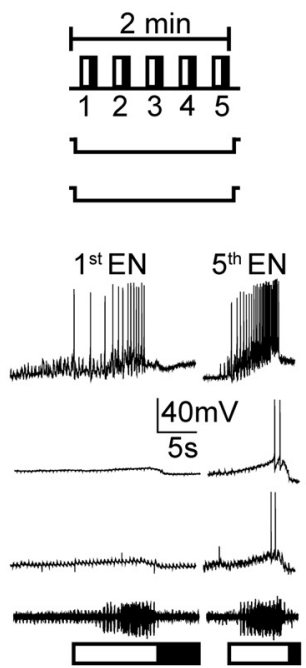

B
$\rightarrow$ no hyperpolarization
$\rightarrow$ hyperpolarization during 5 th
*." hyperpolarization during all

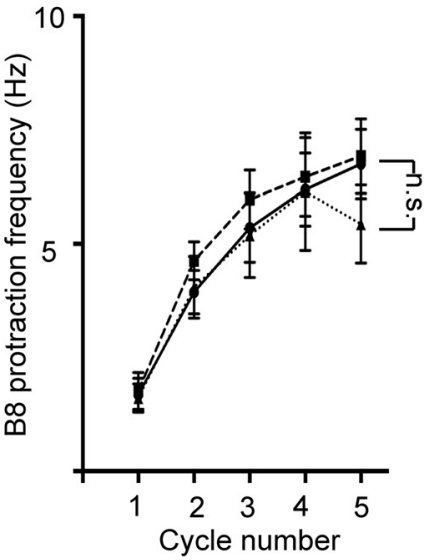

Figure 4. B65 is not necessary for egestive repetition priming. $\boldsymbol{A}$, The EN was stimulated to induce egestive priming under control conditions $(\boldsymbol{A} 1)$, and with $B 65$ bilaterally hyperpolarized, either during the fifth (last) cycle of motor activity (A2), or throughout all five cycles of the motor program (A3). B, Filled circles represent data obtained under control conditions (no B65 hyperpolarization). Filled squares represent data obtained when B65 was hyperpolarized during the fifth cycle. Filled triangles represent data obtained when B65 was hyperpolarized during all cycles. Neither manipulation had a significant effect on the B8 protraction firing frequency during the last cycle $(n=8)$. n.s., Not significant at $p>0.05$. 
A 1. no hyperpolarization

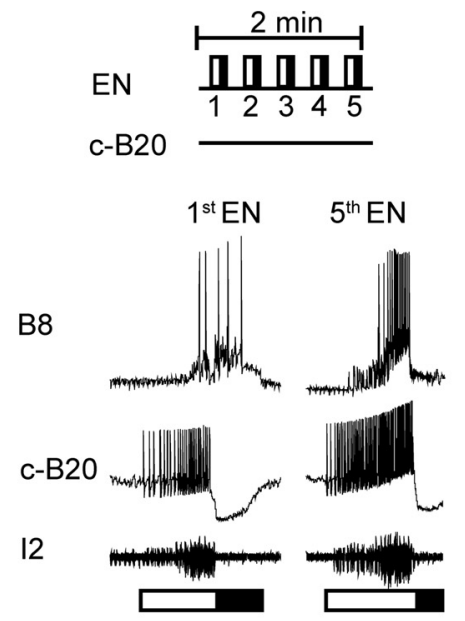

\section{2. hyperpolarization during 5th cycle}
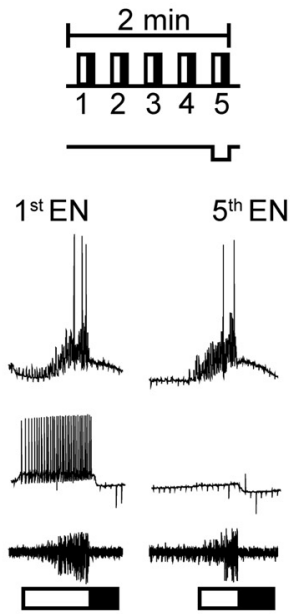

3. hyperpolarization during all cycles

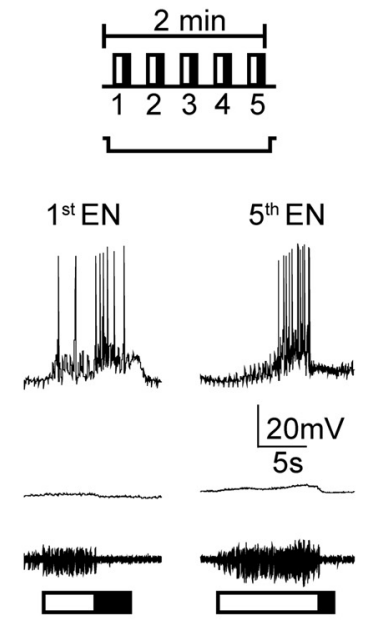

B

- no hyperpolarization

- hyperpolarize during 5th

-." hyperpolarize during all

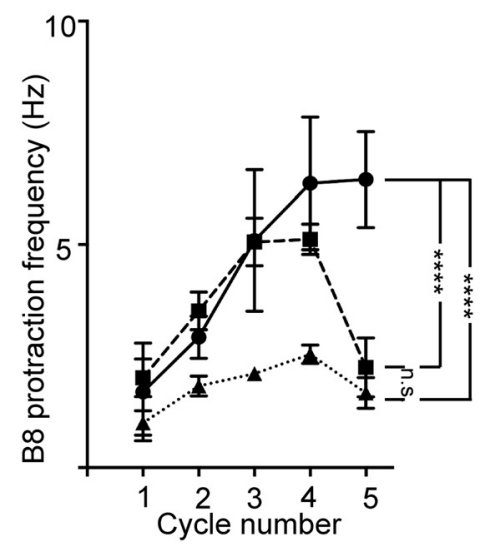

Figure 5. B20 is necessary for egestive repetition priming. $\boldsymbol{A}$, The EN was stimulated to elicit egestive priming under control conditions $(\boldsymbol{A} \mathbf{1})$, and with the contralateral B20 hyperpolarized, either during the fifth (last) cycle of the motor activity (A2), or throughout all five cycles (A3). $\boldsymbol{B}$, Filled circles represent data obtained under control conditions (no B20 hyperpolarization). Filled squares represent data obtained when B20 was hyperpolarized during the fifth cycle. Filled triangles represent data obtained when B20 was hyperpolarized during the all cycles. Hyperpolarization of B20 significantly decreased the B8 firing frequency during the protraction phase of the last cycle $(n=7)$. ${ }^{* * *} p<0.0001$. n.S., Not significant at $p>0.05$.

\section{A}

\section{EN priming}

$$
\text { EN } \frac{2 \text { min }}{12345}
$$

CBI-2
C-B20

cycle 1

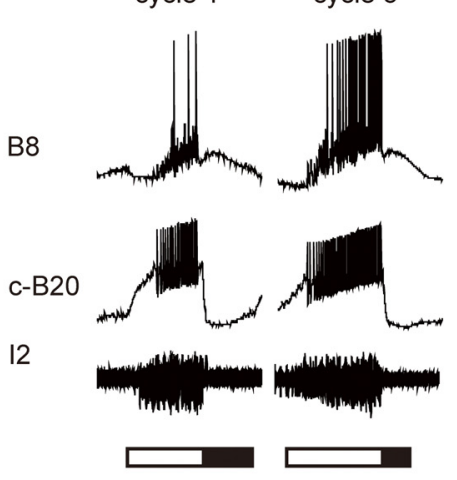

2. positive biasing w/no hyperpolarization

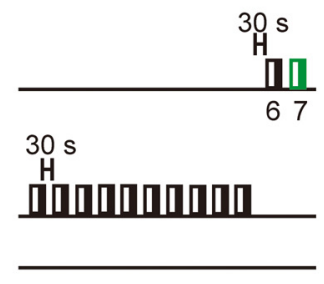

cycle 7

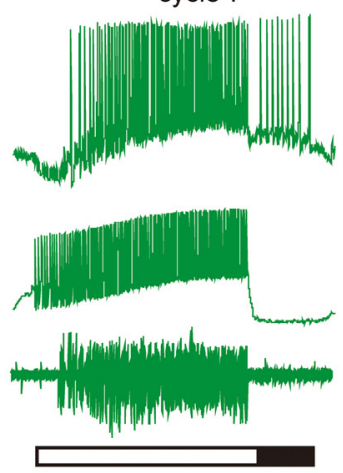

3. positive biasing w/ hyperpolarization

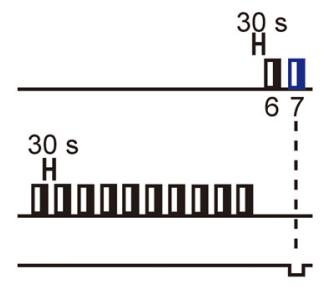

cycle 7
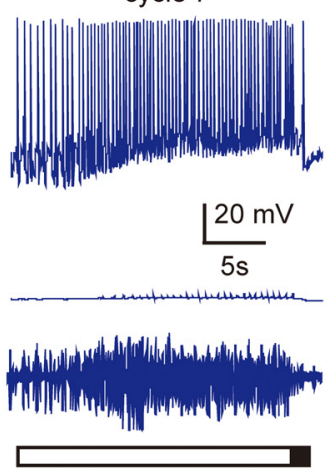

B

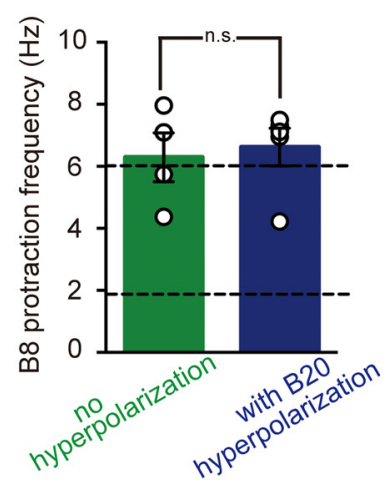

C

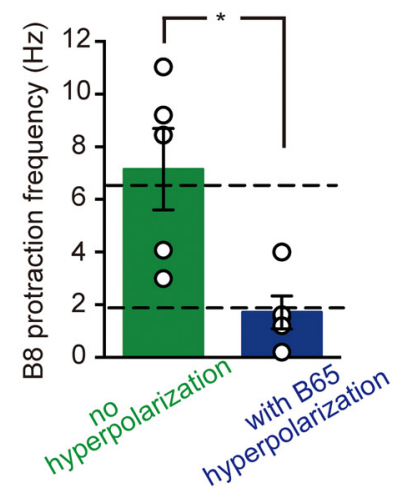

Figure 6. B20 is not essential for positive biasing. $\boldsymbol{A}$, Egestive activity was characterized by inducing egestive repetition priming (A1). Preparations were rested for 30 min, and positive biasing was then induced under control conditions (no B20 hyperpolarization) (A2) or with B20 hyperpolarized (A3). B, The B8 firing frequency after positive biasing during the protraction phase of the seventh cycle of activity with and without B20 hyperpolarization. Bottom and top, Dashed lines plot the B8 firing frequency before and after egestive repetition priming (as shown in $A 1$ ). Bottom, Dashed line indicates the B8 firing frequency during a cycle that is intermediate in nature. Top, Dashed line indicates the B8 firing frequency during a cycle that is egestive. Hyperpolarization of B20 did not decrease the B8 firing frequency (i.e., activity remained egestive) $(n=4)$. C, The B8 firing frequency after positive biasing during the protraction phase of the seventh cycle of activity with and without $\mathrm{B} 65$ hyperpolarization. Hyperpolarization of $B 65$ decreased the B8 firing frequency $(n=5) . A-C$, Green represents data obtained under control conditions (no hyperpolarization). Blue represents data obtained when neurons were hyperpolarized. ${ }^{*} p<0.05$. n.S., Not significant at $p>0.05$. 
A

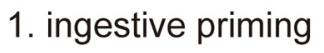

EN

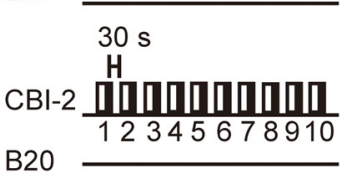

cycle 10

CBI-2

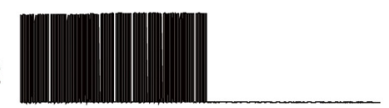

B8

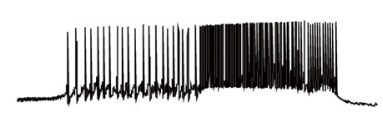

B20

12

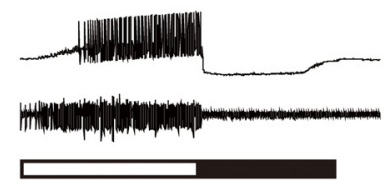

2. negative biasing w/ no hyperpolarization

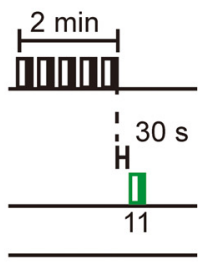

cycle 11

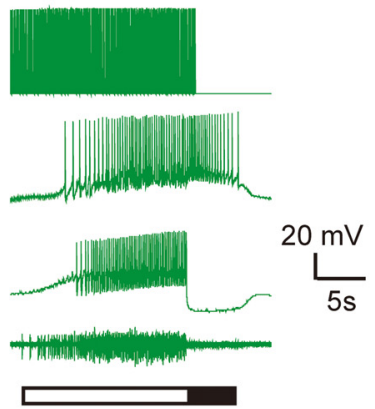

3. negative biasing w/hyperpolarization

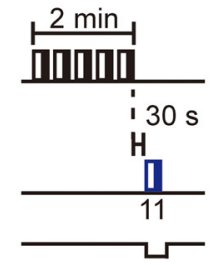

cycle 11
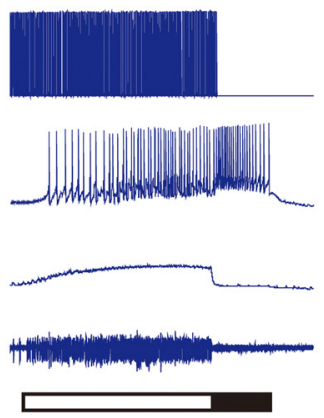

B w/ no hyperpolarization w/ hyperpolarization

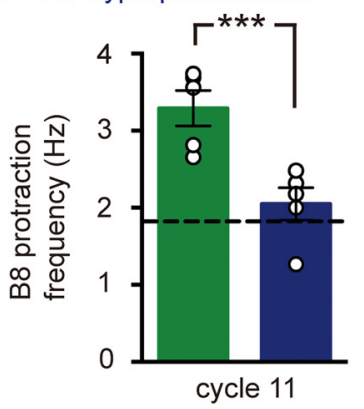

Figure 7. The role of B20 in an egestive-ingestive task switch. $\boldsymbol{A}$, Ingestive activity was characterized by inducing ingestive repetition priming (i.e., 10 cycles of motor activity were triggered by stimulating (BI-2). Preparations were rested for an hour, and then egestive repetition priming was induced. After egestive repetition priming, an attempt was made to switch to ingestive activity under control conditions (no B20 hyperpolarization) (A2) and with B20 hyperpolarized (A3). B, The B8 firing frequency during protraction after ingestive priming with and without B20 hyperpolarization. Dashed line indicates the $\mathrm{B} 8$ firing frequency during the protraction phase of an intermediate cycle of activity triggered by $\mathrm{CBI}-2$. Under control conditions, the B8 firing frequency during protraction is elevated (activity is egestive). This is, however, not the case when B20 is hyperpolarized $(n=5) .{ }^{* *} p<0.001$.

protraction phase of motor programs. Egestive repetition priming and positive biasing are similar in that in both cases this is accomplished via increased activity in a protraction phase interneuron that directly excites B8. The two paradigms differ, however, in that egestive repetition priming requires activity in an interneuron, B20, which is not essential for the generation of a positively biased response. B65 is, however, necessary for generating a positively biased response under conditions where B20 is not. These data indicate that there is degeneracy in the way that egestive activity is patterned in the feeding circuit.

Does degeneracy in the feeding circuit impact task-switching? A further goal of this work was to determine how degeneracy might impact network function.

\section{Hyperpolarization of B20 prevents an egestive-ingestive task switch cost}

Results of the present study together with previous work suggested that differences in the mechanisms used to pattern activity might impact the ability of the network to task switch (i.e., switch to an ingestive configuration). Thus, after egestive repetition priming, there is a "cost" when there is an attempt to switch to ingestive activity (Proekt et al., 2004). More specifically, programs triggered by CBI-2 after egestive repetition priming are egestive (i.e., they are not intermediate in nature as they would be if they were triggered in a quiescent preparation). Previous data suggest that activity in B20 is likely to be at least partially responsible for this switch cost (Proekt et al., 2004, 2007). In particular, increased activity in B20 is likely to be responsible for the increased activity in $\mathrm{B} 8$ during protraction. To determine whether this is the case, we conducted experiments in which we initially established that preparations were capable of generating ingestive activity (i.e., we triggered a series of motor programs using CBI-2) (Fig. 7A1). We then performed egestive-ingestive task switch experiments under control conditions (Fig. 7A2) and with hyperpolarization of B20 (Fig. 7A3). As has been previously reported, programs triggered by CBI-2 after egestive repetition priming were egestive, and the B8 firing frequency during protraction was elevated (Fig. 7A2,B). Hyperpolarization of $\mathrm{B} 20$ produced a significant decrease in protraction phase B8 activity (Fig. $7 A 3, B: t_{(4)}=9.42, p=0.0007$, paired $t$ test; $n=5$ ). When B20 was hyperpolarized, programs were no longer egestive. Further, B8 fired at a frequency that was close to $2 \mathrm{~Hz}$, a frequency that previous work has demonstrated does not evoke a detectable radula movement (Friedman et al., 2009). This indicates that activity in B20 is essential for negative biasing.

In summary, when there is an attempt to trigger ingestive activity after egestive priming, there is a task switch cost. Programs are initially egestive (rather than intermediate). A neuron that impedes task switching (B20) plays a vital role in egestive repetition priming but not in generating a positively biased response. This suggests that switches to ingestive activity might occur more readily after a positively biased response is generated.

\section{The generation of a positively biased response does not} decrease the excitability of the "ingestive" interneuron B40 A third protraction interneuron that is likely to impact task switching is B40. B40 produces a slow, monosynaptic EPSP in B8 that increases the $\mathrm{B} 8$ firing frequency during retraction (Jing and Weiss, 2002). B40 is necessary for CBI-2-induced ingestive activity (i.e., radula closing during retraction) (Jing and Weiss, 2002). When egestive repetition priming is induced, there is a decrease in B40 excitability (Proekt et al., 2007). The impact of generating a positively biased response on B40 excitability has not been determined. A "no effect" result would be predicted if the hypothesis introduced above is correct (i.e., if switches to ingestive 
A

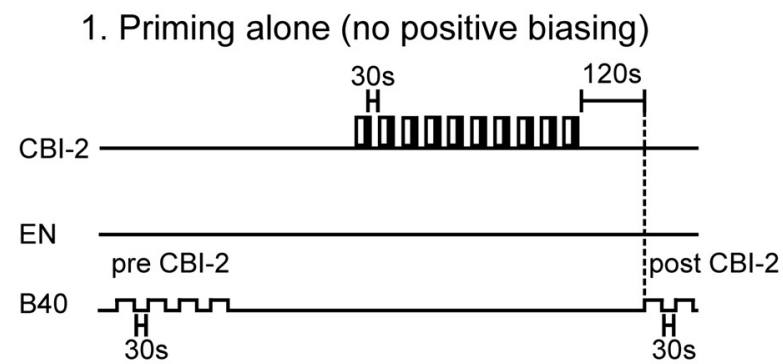

\section{Positive biasing}

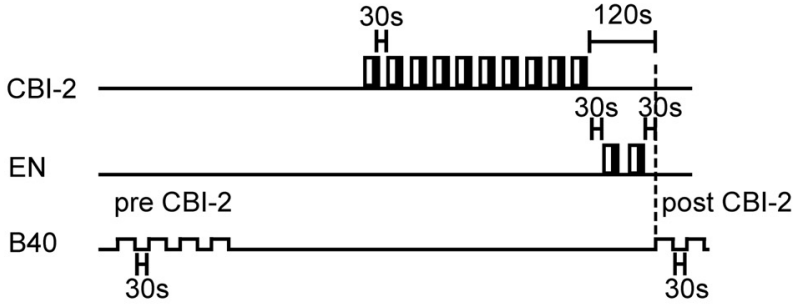

B

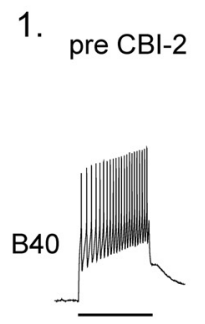

2. pre $\mathrm{CBI}-2$

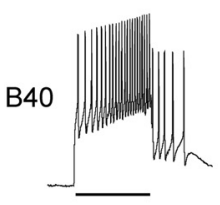

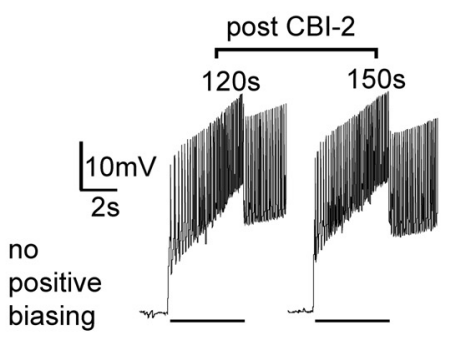
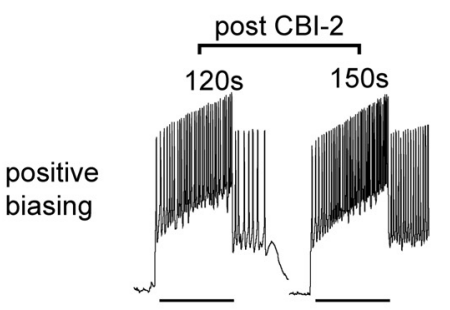

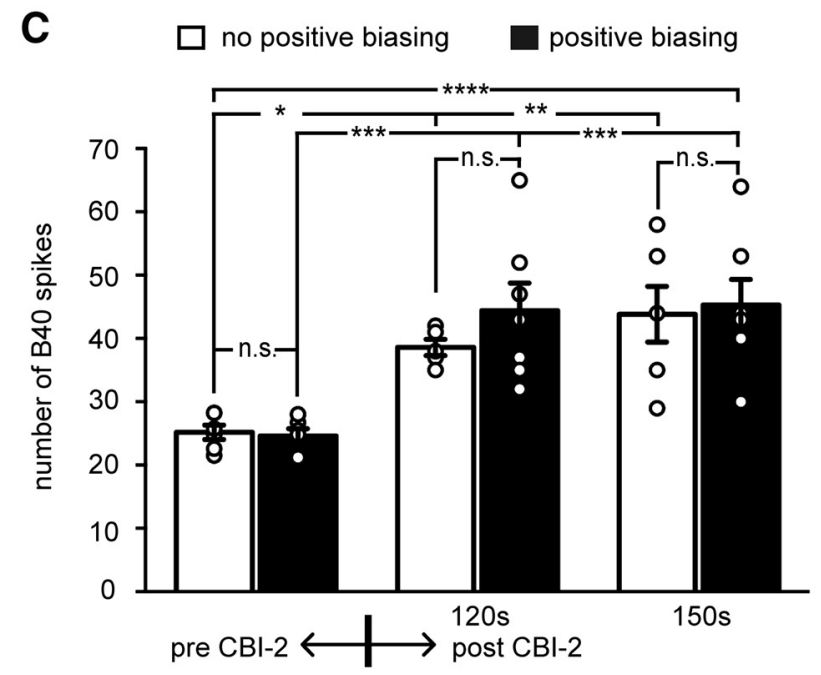

Figure 8. Positive biasing does not decrease the B40 excitability. $A$, Excitability was measured by injecting $4 \mathrm{~s}$ current pulses into B40 before ingestive priming (pre-CBI-2), and after ingestive priming under control conditions (no positive biasing) (A1) and with positive biasing (A2). B, Intracellular recordings from B40. The lines under the traces indicate where current was injected. $\boldsymbol{C}$, The B40 excitability after ingestive priming with (black bars) and without (white bars) subsequent EN stimulation (i.e., positive biasing). Positive biasing did not eliminate the increase in B40 excitability induced by ingestive repetition priming $(n=6) .{ }^{*} p<0.05,{ }^{* *} p<0.01,{ }^{* * *} p<0.001,{ }^{* * * *} p<0.0001$. n.s., Not significant at $p>0.05$.

activity can occur more readily after generating a positively biased response).

To determine how B40 is impacted by generating a positively biased response, we measured its excitability in "rested" preparations (i.e., in preparations in which there was no motor program induction). We also measured excitability after motor programs were triggered using CBI-2 either without (Fig. 8A1) or with (Fig. $8 A 2$ ) subsequent EN stimulation (i.e., positive biasing). CBI-2 stimulation increased excitability in both cases (i.e., there was no effect of positive biasing) (Fig. $8 B, C$; two-way ANOVA: $F_{(2,31)}=$ $19.57 ; p=0.000003$; Sidak's multiple-comparisons post-tests between pre-CBI-2 and post-CBI-2 data; ingestive priming, $p=$ 0.037; positive biasing, $p=0.0005$; Sidak's multiple-comparisons post-test indicated no significant difference between positive biasing and ingestive priming group; at $120 \mathrm{~s}, p=0.67$; at $150 \mathrm{~s}, p=$ $0.99 ; n=6)$. These data indicate that egestive repetition priming and positive biasing differ in their effects on B40. This further suggests that the switch cost observed when there is an attempt to trigger ingestive activity after egestive repetition priming might not be observed after generating a positively biased response.

Ingestive activity can be triggered after generating a positively biased response

In a final set of experiments, we characterized egestive-ingestive switching after positively biased responses were generated to determine whether a switch cost is actually observed. To accomplish this, we induced positively biased responses and then triggered two cycles of activity using CBI-2 (Fig. 9A2). To test for a switch cost, we initially sought to determine whether the CBI-2-induced activity was egestive (i.e., whether the B8 firing frequency during the protraction phase of the motor program was higher than it was when activity was triggered in a similar manner but without the induction of positively biased responses) (Fig. 9A1). The induction of positively biased responses had no significant effect on the B8 firing frequency (Fig. 9B1; program 11: one-way ANOVA with Tukey's post hoc, $p=0.70$; program 12: one-way ANOVA with Tukey's post hoc, $p=0.47$; no positive biasing, $n=5$; positive biasing, $n=4$ ). These data indicate that programs triggered by CBI-2 after the induction of positively biased responses are not egestive.

A further question we addressed was as follows: are programs triggered after positive biasing truly ingestive? In particular, we sought to determine whether positive biasing produced a significant decrease in the firing frequency of B40 during protraction, and/or the firing frequency of B8 during retraction. We found that there was no significant decrease in either case (for the effect on B40, see Fig. 9B3; program 11: $t_{(9)}=1.37, p=0.2, t$ test; program 12: $t_{(9)}=2.2, p=0.06, t$ test; no positive biasing, $n=5$; positive biasing, $n=4$; for the effect on B8, see Figure 9B2; program 11: one-way ANOVA with Tukey's post hoc, $p=0.88$; program 12: one-way ANOVA with Tukey's post hoc, $p=0.38$; no positive biasing, $n=5$; positive biasing, $n=4$ ). These data indicate that positive biasing differs from egestive repetition in that an immediate switch to ingestive activity is possible.

We hypothesized that this difference in the efficacy of task switching was at least in part due to the fact that positive biasing does not decrease the $\mathrm{B} 40$ excitability (as egestive repetition priming does). To determine whether this is the case, we performed experiments in which we attempted egestive-ingestive task switches after the induction of positively biased responses with B40 hyper- 
A

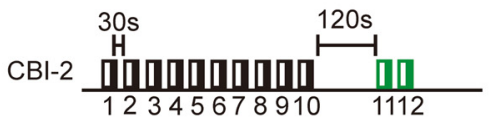

EN

B40

$$
\text { cycle } 11
$$

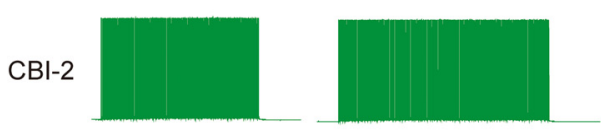

B8
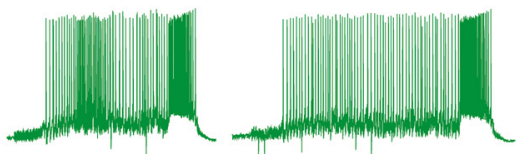

B40

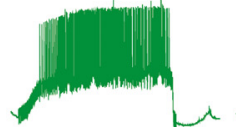

12
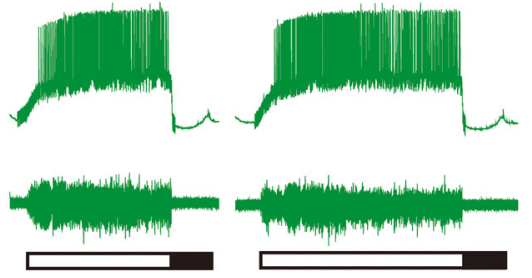

B

1. B8 protraction frequency $(\mathrm{Hz})$

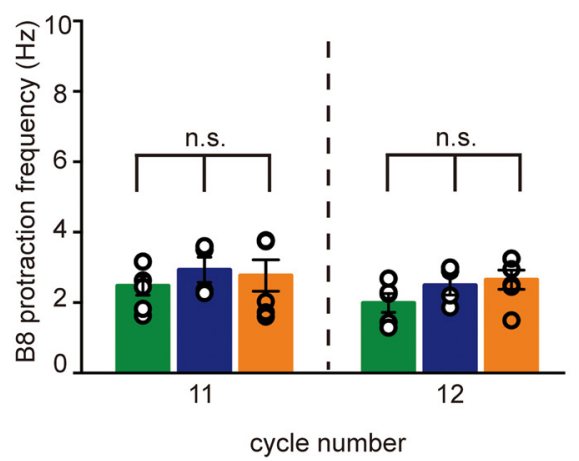

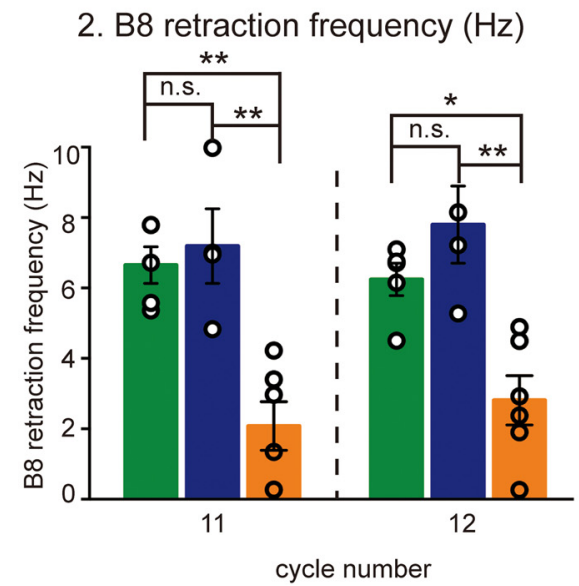

2. positive biasing

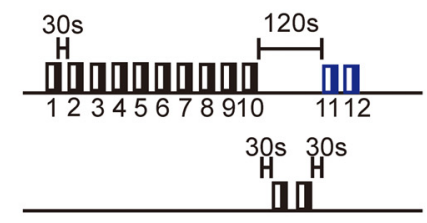

cycle 11

cycle 12
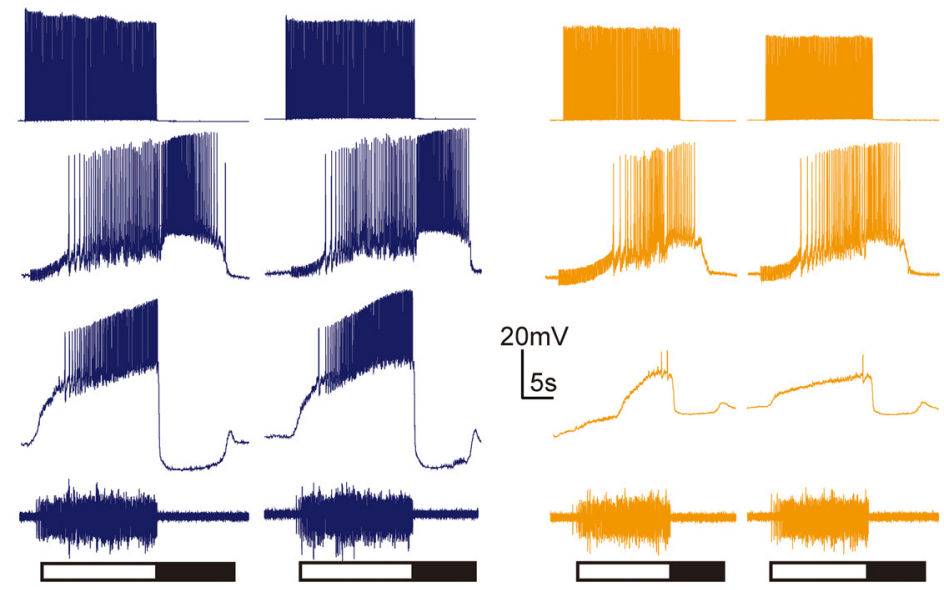

cycle number
3. B40 protraction frequency $(\mathrm{Hz})$

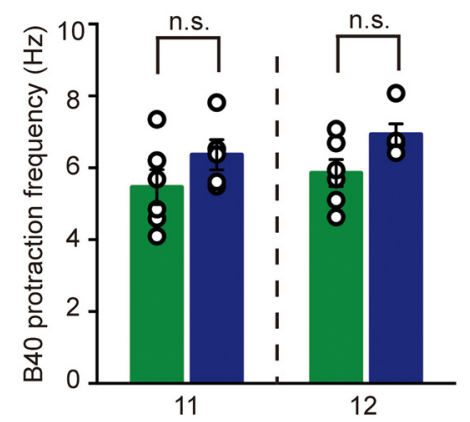

3. positive biasing/B40 hyperpolarization

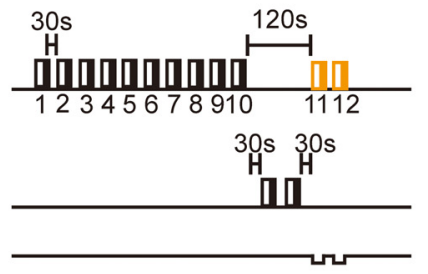

cycle $11 \quad$ cycle 12
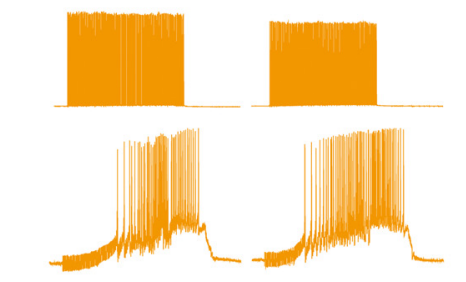

Figure 9. A switch to ingestive activity is possible after positive biasing. A, Two cycles of activity were triggered by stimulating (BI-2 after ingestive repetition priming without positive biasing (A1) and with positive biasing under control condition (no B40 hyperpolarization) (A2), and with B40 hyperpolarized (A3). B1, B8 firing frequency during the protraction phase of the motor program. $B 2$, B8 firing frequency during retraction. $B 3, B 40$ firing frequency during protraction. $B 1-B 3$, No positive biasing, $n=5$; positive biasing, $n=4$; and positive biasing with B40 hyperpolarized, $n=6$. Under control conditions ( $n 0$ B40 hyperpolarization), there was no task switch cost when ingestive activity was induced after positive biasing (i.e., positive biasing did not produce a significant decrease in the B8 firing frequency during the retraction phase of motor programs). This was not the case when B40 was hyperpolarized (B2). ${ }^{*} p<0.05,{ }^{* *} p<0.01$. n.s., Not significant at $p>0.05$.

polarized. We found that B40 hyperpolarization resulted in a significant decrease in the firing frequency of B8 during the retraction phase of the motor program and activity was no longer ingestive (protocol and sample traces shown in Fig. 9A3; Fig. 9B2: program 11: one-way ANOVA with Tukey's post hoc, $p=0.0012$; program 12: one-way ANOVA with Tukey's post hoc, $p=0.0015$; positive biasing, $n=4$; positive biasing with $\mathrm{B} 40$ hyperpolarized, $n=6)$. These data demonstrate that, when B40 is hyperpolarized after the induction of positively biased responses, it is not possible to immediately return to ingestive activity. Together, our data indicate that a functional consequence of degeneracy in the feeding circuit is that task switching is impacted. The changes in the circuit that occur during egestive repetition priming led to an egestive configuration that persists and has a negative impact on the subsequent induction of ingestive activity. In contrast, although the changes in the circuit that occur when positively biased responses are generated also led to an egestive configuration they do not negatively impact subsequent attempts to generate ingestive activity.

\section{Discussion}

We sought to determine whether there is more than one set of circuit parameters that generates egestive activity in the feeding circuit of Aplysia. Activity is egestive when the radula closer motor neurons are primarily active during the protraction phase of the motor program (Morton and Chiel, 1993a, b). Radula closing during protraction pushes food out. We induced egestive activity 


\section{A Task switch after egestive repetition priming}

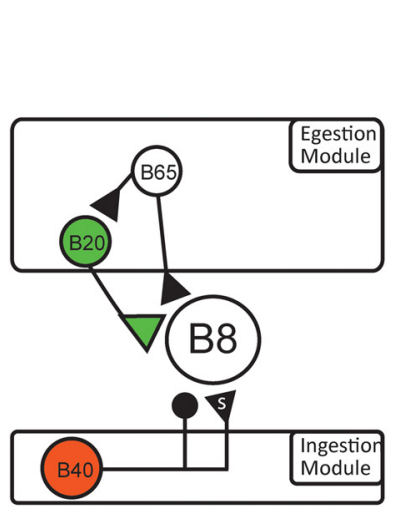

Network changes after repeated EN stimulation
B

Task switch after positive biasing

Figure 10. A, Left, As a result of egestive repetition priming, there is a persistent increase in the excitability of B20 and use-dependent potentiation of B20-B8 synaptic transmission. Additionally, there is a decrease in the excitability of B40. Right, CBI-2 makes a fast excitatory synaptic connection with B20. Consequently, when there is a task switch, B20 fires at an elevated frequency, and the protraction phase firing frequency of $\mathrm{B} 8$ is elevated. Additionally, $\mathrm{CBI}-2$ less effectively activates $\mathrm{B} 40$, and there is relatively little excitatory drive to $\mathrm{B} 8$ during the retraction phase of the motor program. Overall activity is egestive (i.e., negative biasing is observed). B, Left, As a result of positive biasing, the excitability of B65 and B40 is increased. Right, However, B65 is inhibited by CBI-2. Consequently, it does not fire when activity is subsequently triggered by CBI-2 and does not excite B8 during protraction. Additionally, positive biasing does not decrease the B40 excitability. Consequently, it is effectively driven by $C B I-2$ and provides excitatory input to $B 8$ during retraction. Overall activity is ingestive. $A, B$, Green represents an increase in excitability or a potentiation of synaptic transmission. Red represents a decrease in excitability. Filled circles represent inhibitory synaptic connections. Filled triangles represent excitatory connections. A triangle with an " $s$ " represents a slow synaptic connection.

using two paradigms: egestive repetition priming and positive biasing. We showed that circuit parameters differ in the two cases. Thus, we identified an example of degeneracy in the feeding circuit.

\section{Degeneracy in network function in other systems}

Degeneracy in network function has been demonstrated in other species. A well-characterized system where it has been studied is the crustacean stomatogastric nervous system. Here the potential for degeneracy was strikingly illustrated in a computational study that simulated millions of versions of a triphasic motor program (Prinz et al., 2004). This study demonstrated that virtually indistinguishable activity patterns could arise from widely disparate sets of circuit parameters. That degeneracy is seen in the biological stomatogastric network was subsequently confirmed. Variability of ion channel expression was measured using voltage-clamp techniques to measure currents, and single-cell PCR to measure channel mRNA expression (e.g., Schulz et al., 2006). These studies demonstrated that both conductances and mRNA levels vary considerably. Nevertheless, uniquely identifiable neurons have almost superimposable activity patterns (Schulz et al., 2006).

Other model organisms in which network degeneracy has been reported include the leech and C. elegans. For example, in the leech, there is variability in the strength of synaptic connections that control a stereotyped behavior, heartbeat (Norris et al., 2007; Roffman et al., 2012). In C. elegans, cell ablation and rescue experiments have demonstrated that different combinations of thermosensory neurons are necessary and sufficient for negative thermotaxis under different conditions (Beverly et al., 2011). Further, pharyngeal pumping can be triggered by more than one motor neuron (Trojanowski et al., 2014).

\section{Functional significance of network degeneracy}

Although network degeneracy is observed in a number of species, it is not clear why this is the case. It is possible that it is beneficial.
Alternatively, it could be a reflection of the fact that, although it is important for a network to be able to generate a particular output, it is not important how this is achieved. In support of the idea that it is beneficial, others have argued that degeneracy makes circuit function more robust by providing multiple elements that can be varied to arrive at the same endpoint (for further discussion see Cropper et al., 2016). Our data also support the idea that degeneracy has functional significance. However, we present a novel idea that postulates that it can have consequences for statedependent network dynamics. We suggest that the method used to pattern neural activity can determine how readily activity is reconfigured. Together with previous work (Proekt et al., 2004), our data indicate that when egestive activity is generated by egestive repetition priming, the feeding network cannot be immediately reconfigured to generate an ingestive motor program. In contrast, we show that task switching can occur more readily if egestive activity is generated using the positive biasing paradigm.

\section{Circuit parameters that promote egestive-ingestive task switching in the feeding network}

These and previous results identify circuit parameters that uniquely define egestive repetition priming as opposed to positive biasing. For example, increased synaptic input to the B8 radula closer motor neurons during protraction primarily results from input from two different interneurons (B20 for repetition priming and B65 for positive biasing). This difference partially accounts for the difference in task switching observed. In particular, it accounts for the negative biasing that is observed after egestive repetition priming but not positive biasing. As egestive repetition priming develops, there is an increase in B20's excitability and therefore its firing frequency (Fig. 10A). Effects of egestive repetition priming on B20 are at least partially peptide-mediated and persist (Wu et al., 2010). For example, after EN stimulation, the B20 excitability does not immediately return to baseline levels (Proekt et al., 2007). Further, during egestive repetition priming, 
persistent potentiation of B20-B8 synaptic transmission occurs (Proekt et al., 2004, 2007). These changes have consequences when there is an attempt to task switch because there is a fast excitatory synaptic connection between CBI-2 and B20 (Fig. 10A, right) (Jing and Weiss, 2001). In an unprimed network, this connection is weak. However, after EN stimulation, it becomes more effective. Consequently, the CBI-2-induced increase in B20 activity leads to an increase in the $\mathrm{B} 8$ firing frequency during protraction, and programs are egestive. In this situation, there is a switch cost since CBI-2 triggers a response that is less behaviorally appropriate than the response that is triggered in a quiescent preparation.

When egestive activity is generated using the positive biasing protocol, increases in $\mathrm{B} 8$ activity during protraction are primarily mediated by input from B65. B65 and B20 differ in an interesting way. $\mathrm{B} 65$ is inhibited, rather than excited, by CBI-2 (Fig. $10 \mathrm{~A}$ vs Fig. 10B) (Jing and Weiss, 2005). Consequently, excitability increases that develop during positive biasing do not have a negative impact when there is an attempt to make an egestiveingestive task switch (Fig. 10B, right). In this situation, there is no switch cost. In summary, egestive repetition priming and positive biasing are both mediated by an increase in the excitability of an "egestive" interneuron. In one case (egestive repetition priming), the "egestive" interneuron also receives excitatory input from the ingestive CPG input. Consequently, negative biasing is observed. In the other case (positive biasing), the egestive interneuron is inhibited when the ingestive CPG input is activated. Consequently, there is no negative biasing.

Differential B20 versus B65 activation can account for the fact that programs triggered by CBI-2 after priming are egestive, whereas programs triggered after the induction of positively biased results are generated are not. However, it cannot fully account for the fact that programs triggered after positive biasing are more than "not egestive" (i.e., they are truly ingestive). A "not egestive" program is one in which the B8 firing frequency during protraction is relatively low (i.e., the radula would not close as it moves forward), and food would not be pushed out of the buccal cavity. If a program is truly ingestive, relatively high-frequency B8 activity during retraction is necessary. Food is not pulled into the buccal cavity unless the radula closes as it retracts. B20 and B65 are both active during protraction and make fast excitatory connections with B8 (Teyke et al., 1993; Kabotyanski et al., 1998; Jing and Weiss, 2001, 2005). Consequently, these interneurons do not have a major impact on $\mathrm{B} 8$ during retraction.

We demonstrate that egestive repetition priming and positive biasing also differ in their effects on B40. B40 makes a complex synaptic connection with B8 (Jing and Weiss, 2002). B40 is active during protraction, but the excitatory component of the induced PSP is slow and primarily impacts B8 during retraction (Jing and Weiss, 2002). Interestingly, egestive repetition priming and positive biasing do not have the same effect on B40 excitability. Egestive repetition priming decreases it (Fig. 10A), but positive biasing does not (Fig. 10B). Consequently, when ingestive activity is triggered after positive biasing, B40 activity remains elevated (Fig. 10B, right). Our hyperpolarization experiments indicate that this contributes to the synaptic drive that $\mathrm{B} 8$ receives during retraction. In conclusion, we have identified several circuit parameters that differ when egestive activity is triggered via egestive repetition priming versus positive biasing. Further, we have demonstrated that these differences have consequences for task switching.

\section{What is the functional significance of the difference in task switching?}

An additional question is as follows: why would there be differences in task switching in the two situations? Egestive repetition priming presumably occurs when maintained exposure to an "egestive" stimulus triggers repeated rejection responses. Under physiological conditions, this is likely to occur during satiation, or in the presence of a noxious substance. If so, a sudden switch to ingestive activity may be counterproductive (e.g., a noxious substance could be accidently ingested). Thus, negative biasing may be important to prevent this from occurring. In contrast, positively biased responses will be generated immediately after there has been repeated exposure to an "ingestive" stimulus (food). Under physiological conditions, this type of rapid ingestive/egestive switch has been observed when animals that have been consuming a strip of seaweed suddenly encounter resistance (e.g., the other end of the seaweed is attached to a substrate) (Proekt et al., 2008). The "attached" piece of seaweed that cannot be ingested is egested. In this situation, a rapid return to ingestive activity is presumably beneficial since it allows animals to try again (i.e., to ingest a new strip). In summary, we suggest that network degeneracy makes it possible for animals to make relatively gradual egestive/ingestive task switches under one set of circumstances and relatively rapid switches under another. Thus, we suggest that the ability of a network to generate activity using more than one set of circuit parameters increases its potential for flexibility.

\section{References}

Beverly M, Anbil S, Sengupta P (2011) Degeneracy and neuromodulation among thermosensory neurons contribute to robust thermosensory behaviors in Caenorhabditis elegans. J Neurosci 31:11718-11727.

Church PJ, Lloyd PE (1994) Activity of multiple identified motor neurons recorded intracellularly during evoked feedinglike motor programs in Aplysia. J Neurophysiol 72:1794-1809.

Cropper EC, Dacks AM, Weiss KR (2016) Consequences of degeneracy in network function. Curr Opin Neurobiol 41:62-67.

Dacks AM, Weiss KR (2013) Latent modulation: a basis for non-disruptive promotion of two incompatible behaviors by a single network state. J Neurosci 33:3786-3798.

Friedman AK, Weiss KR (2010) Repetition priming of motoneuronal activity in a small motor network: intercellular and intracellular signaling. J Neurosci 30:8906-8919.

Friedman AK, Zhurov Y, Ludwar BC, Weiss KR (2009) Motor outputs in a multitasking network: relative contributions of inputs and experiencedependent network states. J Neurophysiol 102:3711-3727.

Golowasch J (2014) Ionic current variability and functional stability in the nervous system. Bioscience 64:570-580.

Hurwitz I, Susswein AJ (1996) B64, a newly identified central pattern generator element producing a phase switch from protraction to retraction in buccal motor programs of Aplysia californica. J Neurophysiol 75:13271344.

Hurwitz I, Perrins R, Xin Y, Weiss KR, Kupfermann I (1999) C-PR neuron of Aplysia has differential effects on 'feeding' cerebral interneurons, including myomodulin-positive CBI-12. J Neurophysiol 81:521-534.

Jing J, Weiss KR (2001) Neural mechanisms of motor program switching in Aplysia. J Neurosci 21:7349-7362.

Jing J, Weiss KR (2002) Interneuronal basis of the generation of related but distinct motor programs in Aplysia: implications for current neuronal models of vertebrate intralimb coordination. J Neurosci 22:6228-6238.

Jing J, Weiss KR (2005) Generation of variants of a motor act in a modular and hierarchical motor network. Curr Biol 15:1712-1721.

Jing J, Cropper EC, Hurwitz I, Weiss KR (2004) The construction of movement with behavior-specific and behavior-independent modules. J Neurosci 24:6315-6325.

Kabotyanski EA, Baxter DA, Byrne JH (1998) Identification and characterization of catecholaminergic neuron B65, which initiates and modifies patterned activity in the buccal ganglia of Aplysia. J Neurophysiol 79:605-621. 
Kupfermann I (1974) Feeding behavior in Aplysia: a simple system for the study of motivation. Behav Biol 10:1-26.

Kuslansky B, Weiss KR, Kupfermann I (1987) Mechanisms underlying satiation of feeding-behavior of the mollusk Aplysia. Behav Neural Biol 48:278-303.

Lamb DG, Calabrese RL (2013) Correlated conductance parameters in leech heart motor neurons contribute to motor pattern formation. PLoS One 8:e79267.

Marder E, Goeritz ML, Otopalik AG (2015) Robust circuit rhythms in small circuits arise from variable circuit components and mechanisms. Curr Opin Neurobiol 31:156-163.

Morgan PT, Perrins R, Lloyd PE, Weiss KR (2000) Intrinsic and extrinsic modulation of a single central pattern generating circuit. J Neurophysiol 84:1186-1193.

Morgan PT, Jing J, Vilim FS, Weiss KR (2002) Interneuronal and peptidergic control of motor pattern switching in Aplysia. J Neurophysiol 87:49-61.

Morton DW, Chiel HJ (1993a) In vivo buccal nerve activity that distinguishes ingestion from rejection can be used to predict behavioral transitions in Aplysia. J Comp Physiol A 172:17-32.

Morton DW, Chiel HJ (1993b) The timing of activity in motor neurons that produce radula movements distinguishes ingestion from rejection in Aplysia. J Comp Physiol A 173:519-536.

Nargeot R, Baxter DA, Byrne JH (1999a) In vitro analog of operant conditioning in Aplysia: I. Contingent reinforcement modifies the functional dynamics of an identified neuron. J Neurosci 19:2247-2260.

Nargeot R, Baxter DA, Byrne JH (1999b) In vitro analog of operant conditioning in Aplysia: II. Modifications of the functional dynamics of an identified neuron contribute to motor pattern selection. J Neurosci 19:2261-2272.

Norris BJ, Weaver AL, Wenning A, García PS, Calabrese RL (2007) A central pattern generator producing alternative outputs: pattern, strength, and dynamics of premotor synaptic input to leech heart motor neurons. J Neurophysiol 98:2992-3005.

Perkins MH, Cropper EC, Weiss KR (2018) Cellular effects of repetition priming in the Aplysia feeding network are suppressed during a taskswitch but persist and facilitate a return to the primed state. J Neurosci 38:6475-6490.
Prinz AA, Bucher D, Marder E (2004) Similar network activity from disparate circuit parameters. Nat Neurosci 7:1345-1352.

Proekt A, Brezina V, Weiss KR (2004) Dynamical basis of intentions and expectations in a simple neuronal network. Proc Natl Acad Sci U S A 101:9447-9452.

Proekt A, Jing J, Weiss KR (2007) Multiple contributions of an inputrepresenting neuron to the dynamics of the Aplysia feeding network. J Neurophysiol 97:3046-3056.

Proekt A, Wong J, Zhurov Y, Kozlova N, Weiss KR, Brezina V (2008) Predicting adaptive behavior in the environment from central nervous system dynamics. PLoS One 3:e3678.

Roffman RC, Norris BJ, Calabrese RL (2012) Animal-to-animal variability of connection strength in the leech heartbeat central pattern generator. J Neurophysiol 107:1681-1693

Rosen SC, Teyke T, Miller MW, Weiss KR, Kupfermann I (1991) Identification and characterization of cerebral-to-buccal interneurons implicated in the control of motor programs associated with feeding in Aplysia. J Neurosci 11:3630-3655.

Sánchez JA, Kirk MD (2001) Cerebral-buccal pathways in Aplysia californica: synaptic connections, cooperative interneuronal effects and feedback during buccal motor programs. J Comp Physiol A 187:801-815.

Schulz DJ, Goaillard JM, Marder E (2006) Variable channel expression in identified single and electrically coupled neurons in different animals. Nat Neurosci 9:356-362.

Siniscalchi MJ, Cropper EC, Jing J, Weiss KR (2016) Repetition priming of motor activity mediated by a central pattern generator: the importance of extrinsic vs intrinsic program initiators. J Neurophysiol 116:1821-1830.

Teyke T, Rosen SC, Weiss KR, Kupfermann I (1993) Dopaminergic neuron B20 generates rhythmic neuronal activity in the feeding motor circuitry of Aplysia. Brain Res 630:226-237.

Trojanowski NF, Padovan-Merhar O, Raizen DM, Fang-Yen C (2014) Neural and genetic degeneracy underlies Caenorhabditis elegans feeding behavior. J Neurophysiol 112:951-961.

Wu JS, Vilim FS, Hatcher NG, Due MR, Sweedler JV, Weiss KR, Jing J (2010) Composite modulatory feedforward loop contributes to the establishment of a network state. J Neurophysiol 103:2174-2184. 